\title{
Five-day regimen of intramuscular or subcutaneous self-administered adrenocorticotropic hormone gel for acute exacerbations of multiple sclerosis: a prospective, randomized, open-label pilot trial
}

This article was published in the following Dove Press journal:

Drug Design, Development and Therapy

8 July 2011

Number of times this article has been viewed

\section{James P Simsarian \\ Carol Saunders \\ D Michelle Smith}

Neurology Center of Fairfax Ltd, Fairfax, VA, USA
Correspondence: James P Simsarian Neurology Center of Fairfax Ltd, 3020 Hamaker Court, Suite 400, Fairfax, VA 2203I-2220, USA

$\mathrm{Tel}+\mathrm{I} 703876080 \mathrm{I}$

Fax +I 7038760832

Email jsimsarian@neurologyfairfax.com
Background: Despite over 50 years of experience with adrenocorticotropic hormone (ACTH) as a treatment for acute exacerbations of multiple sclerosis, there have been no trials examining the options of the 2-3-week dosing regimen or intramuscular injection protocol used in the original trials. At our clinic, we performed a small, prospective, randomized pilot study to examine the efficacy and safety of, and patient satisfaction with, a short (five-day) self-administered ACTH dosing protocol for exacerbations of multiple sclerosis, and to compare the subcutaneous and intramuscular routes of administration.

Methods: Patients for this study were recruited from an outpatient treatment clinic. Each patient self-administered natural ACTH gel $80 \mathrm{U} /$ day by subcutaneous or intramuscular injection for five consecutive days and was evaluated at baseline and on days 7 and 14. Patient feedback was collected using the Patient Global Impression of Change (PGI-C, the primary efficacy measure), a patient global visual analog scale, the Expanded Disability Status Scale, a timed walk, the Nine-hole Peg Test, and the Clinical Global Impression of Change.

Results: Of the 20 enrolled patients (mean age 39.5 years), 19 completed the study. On day $14,61.1 \%$ of patients (11 of 18 with day 14 scores) were treatment responders, and rated their condition as "very much improved" or "much improved" on the PGI-C. The intramuscular group had numerically more responders, but there was no significant difference in the proportion of responders between the intramuscular and subcutaneous groups at day $14(P=0.3)$. The intramuscular route of injection was associated with more injection site pain than the subcutaneous route.

Conclusion: A shorter five-day course of intramuscular or subcutaneous ACTH gel may improve symptoms associated with acute exacerbations of multiple sclerosis. Larger studies with standard of care controls are needed to confirm whether this shorter course of intramuscular or subcutaneous ACTH gel is effective and could potentially be substituted for the standard 14-day treatment.

Keywords: adrenocorticotropic hormone, ACTH, multiple sclerosis, acute exacerbations, Acthar ${ }^{\circledR}$ Gel, autoimmune diseases

\section{Introduction}

Multiple sclerosis is a chronic progressive disease resulting in damage to the myelin sheath and nerves of the central nervous system (brain, spinal cord, and optic nerves). It is thought to be an autoimmune disorder, is usually diagnosed during early to late 
adulthood, and affects 2-3 times more women than men. ${ }^{1}$ Approximately 350,000 Americans are diagnosed with multiple sclerosis, ${ }^{2}$ although the number may be higher because symptoms generally do not appear until the disease has progressed.

Symptoms and their severity and duration vary greatly among patients. Symptoms may include extreme fatigue, difficulty with memory and concentration, vision loss, blurred or double vision, numbness, tingling, loss of balance and coordination, tremors, stiffness, slurred speech, and bladder/ bowel dysfunction. ${ }^{1,2}$ Multiple sclerosis is rarely fatal, and the majority of patients do not become severely disabled. The most common form of multiple sclerosis is relapsingremitting ( $85 \%$ of patients), where patients experience discrete and acute exacerbations or flareups of symptoms, followed by partial or complete remission from symptoms or disease activity. ${ }^{2}$

Current treatments for multiple sclerosis include diseasemodifying drugs that reduce the frequency and intensity of exacerbations, and drugs that treat the symptoms or the exacerbation itself. ${ }^{1,2}$ The standard treatment for severe exacerbations is a 3-5-day course of intravenous methylprednisolone. If intravenous corticosteroids cannot be administered, are unavailable, are not effective, or are not well tolerated, adrenocorticotropic hormone (ACTH) is recommended as an alternative option. ${ }^{2}$

ACTH has demonstrated efficacy in the treatment of acute exacerbations of multiple sclerosis in controlled clinical trials, and is approved by the US Food and Drug Administration for treatment for acute exacerbations of multiple sclerosis. ${ }^{3-5}$ In all trials of ACTH in multiple sclerosis, the drug was administered as intramuscular injections for at least two weeks. ${ }^{3-5}$ Based on these trials, the protocol currently approved by the Food and Drug Administration prescribes daily doses of ACTH ranging from 80 to $120 \mathrm{U}$, given as intramuscular or subcutaneous injections for 14-21 days to treat acute exacerbations of multiple sclerosis. ${ }^{6}$ However, a shorter protocol may be effective. One clinical trial of ACTH, for example, found symptoms significantly improved compared with placebo after seven days of treatment. ${ }^{4}$ Similarly, methylprednisolone, the standard therapy for treatment of exacerbations, was originally given as a longer course of treatment before it was reported that a shorter five-day course was equally effective. ${ }^{7}$

In many instances, subcutaneous injections may offer a viable route of administration for drugs traditionally administered intramuscularly. It has recently been shown that subcutaneous and intramuscular injections of
$\mathrm{ACTH}$ are bioequivalent in stimulating cortisol levels in healthy volunteers. ${ }^{8}$ The subcutaneous injection is easier to administer for the following reasons, ie, a larger injectable area, no need to identify anatomical landmarks, easier to administer in emaciated patients, and a superior safety profile. ${ }^{9}$ Because the gel formulation of natural ACTH has been approved to be dosed subcutaneously for acute exacerbations of multiple sclerosis and for other indications, and shorter courses of treatment are suggested by the literature, we conducted a pilot study to test a shorter, self-administered five-day injection regimen and to compare the effectiveness, safety, and patient satisfaction of these two routes of ACTH administration in patients with an acute exacerbation of multiple sclerosis.

\section{Materials and methods Patients}

Study participants were men and women aged $\geq 18$ years with a clinical diagnosis of relapsing-remitting, secondary progressive, or progressive relapsing multiple sclerosis, who presented at an outpatient clinic with an acute exacerbation within 24-120 hours of symptom onset. While discussing treatment options, participants were given the option to be screened for the study by the investigator. Those who chose not to participate were given the standard treatment of intravenous methylprednisolone. Patients who were on other investigative drugs, chemotherapeutic agents, the humanized monoclonal antibody, natalizumab (Tysabri ${ }^{\circledR}$, Biogen Idec, Cambridge, MA), or had a history of a clinically significant infection within 30 days prior to study entry, were excluded. Women who were nursing, pregnant, or planning to become pregnant, and those of childbearing potential (ie, those who were not postmenopausal for at least one year) who were unwilling to use effective contraception were excluded. Concomitant use of certain medications (acetylsalicylic acid, anticholinesterases, oral anticoagulants, chemotherapeutic agents, cholestyramine, systemic corticosteroids, cyclosporine, digitalis glycosides, ephedrine, ketoconazole, macrolide antibiotics, phenobarbital, phenytoin, rifampicin, thalidomide, or vaccines) was not permitted during the study. Informed consent was signed and collected by either the investigating physician or by staff after the patient had the opportunity to learn and ask questions about the study. Patients were treated immediately after being diagnosed and providing informed consent.

\section{Study design, randomization, and dosing}

This was a two-week prospective, randomized, openlabel, single-center, pilot study with two treatment arms 


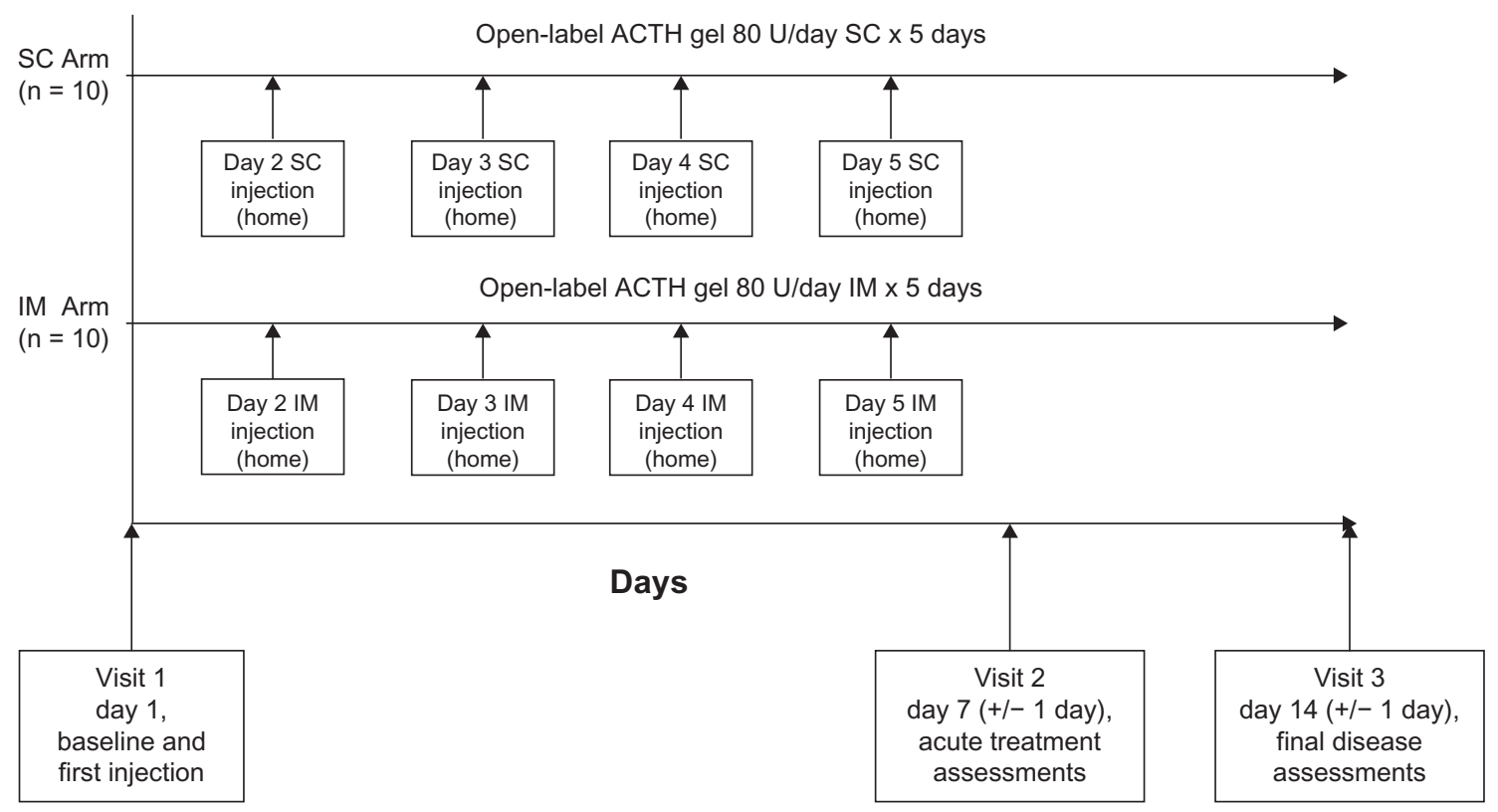

Figure I Study design. Study subjects were randomized to self-administer either $80 \mathrm{U}$ of intramuscular ACTH gel or $80 \mathrm{U}$ of subcutaneous ACTH gel daily on days I-5 of the study.

Abbreviations: IM, intramuscular; SC, subcutaneous; ACTH, adrenocorticotropic hormone.

(see Figure 1). A five-day treatment course was chosen because the standard corticosteroid intravenous treatment is 3-5 days. The dose of $80 \mathrm{U} /$ day is the lower end of the approved dose range for acute exacerbations of multiple sclerosis. Since this is a self-administration study, we chose the lower dose $(80 \mathrm{U} /$ day) because each multiuse vial of ACTH holds $80 \mathrm{U} / \mathrm{mL}$ in $5 \mathrm{~mL}$ gel volume, and we believe this offers the most convenient and cost-effective dosing for the patient without the need to discard any unused drug at the end of treatment.

After clinical evaluation and informed consent was obtained at the first visit on day 1 , subjects were randomized using a random-number generator in a 1:1 scheme to receive ACTH gel (HP Acthar ${ }^{\circledR}$ gel [repository corticotrophin injection], Questcor Pharmaceuticals, Union City, $\mathrm{CA})^{6}$ dosed at $80 \mathrm{U} /$ day, given as either an intramuscular or subcutaneous injection on days $1-5$ of the study. Participants were trained to self-administer the injection by study personnel during the first visit. During the study period, ACTH gel injections were the only means of treating the exacerbations. The subjects were then reevaluated on days 7 and 14. Compliance was evaluated by daily responses on the patient treatment performance surveys.

\section{Outcomes}

\section{Treatment performance}

Both subjective and objective measures of drug response were used in the study (Table 1). The primary outcome measure was the Patient Global Impression of Change (PGI-C), a selfadministered daily change scale, with scores ranging from 1 (very much improved) to 7 (very much worse) used to record a patient's impression about the effectiveness of the treatment. ${ }^{10}$ The measure of symptom alleviation was the final time point score of the PGI-C on day 14; patients reporting change scores of 1 and 2 were considered to be treatment responders.

A patient visual analog scale was also used to quantify symptoms associated with their acute exacerbation of multiple sclerosis. Each day during the course of the study, participants marked a linear scale that ranged from 1 (no acute exacerbation of symptoms) to 10 (worst imaginable acute exacerbation of symptoms). In addition, we assessed patient satisfaction with the self-administration technique using a drug attitude questionnaire developed for this study.

The clinical impression of treatment performance with intramuscular or subcutaneous ACTH gel was quantified using a Clinical Global Impression of Change (CGI-C) questionnaire. ${ }^{11}$ Disability was quantified using the Kurtzke Expanded Disability Status Scale (EDSS) at each study visit on days 1,7 , and $14 .{ }^{12}$ Objective measures of drug performance included documentation of results of a timed walk ${ }^{13}$ and the Nine-hole Peg Test for finger dexterity, ${ }^{14}$ which were measured pretreatment (on day 1) and on days 7 and 14 after treatment.

\section{Safety}

Medical history was elicited, and physical examination, including vital signs, was performed on all participants at study 
Table I Schedule of assessments

\begin{tabular}{|c|c|c|c|c|c|c|c|c|c|c|c|c|c|c|}
\hline & \multicolumn{14}{|c|}{ Day } \\
\hline & $\mathbf{I}$ & 2 & 3 & 4 & 5 & 6 & 7 & 8 & 9 & 10 & II & 12 & 13 & 14 \\
\hline Self-injection & $x$ & $x$ & $x$ & $x$ & $x$ & & & & & & & & & \\
\hline \multicolumn{15}{|c|}{ Subjective efficacy assessments } \\
\hline Patient visual analog scale & $x$ & $x$ & $x$ & $x$ & $x$ & $x$ & $x$ & $x$ & $x$ & $x$ & $x$ & $x$ & $x$ & $x$ \\
\hline PGI-C & & $x$ & $x$ & $x$ & $x$ & $x$ & $x$ & $x$ & $x$ & $x$ & $x$ & $x$ & $x$ & $x$ \\
\hline Drug attitude questionnaire & & & & & & & $x$ & & & & & & & $x$ \\
\hline \multicolumn{15}{|c|}{ Objective efficacy assessments } \\
\hline CGI-C & & & & & & & $x$ & & & & & & & $x$ \\
\hline Nine-hole Peg Test & $x$ & & & & & & $x$ & & & & & & & $x$ \\
\hline Timed walk & $x$ & & & & & & $x$ & & & & & & & $x$ \\
\hline EDSS & $x$ & & & & & & $x$ & & & & & & & $x$ \\
\hline
\end{tabular}

Abbreviations: EDSS, Expanded Disability Status Scale; CGI-C, Clinical Global Impression of Change; PGI-C, Patient Global Impression of Change.

entry (day 1) and at the two follow-up visits (days 7 and 14). Complete blood counts and urinalysis were performed on all three study visits. All adverse events occurring during the study period were documented. Serious adverse events were classified as those that required inpatient hospitalization, were life-threatening, or resulted in significant disability or death.

\section{Data analysis}

All participants receiving at least one dose of medication were included in the safety analysis; subjects receiving at least one dose of study medication and completing one PGI-C scale were included in the treatment performance analysis. Descriptive statistics were used for treatment performance and safety analyses. This study was designed neither with a formal hypothesis nor with power calculations.

\section{Results}

\section{Study population}

A total of 20 patients were enrolled in this pilot study, comprising 17 women (85\%) and three men (15\%). All participants were Caucasian, and aged 25-59 (mean 39.5 \pm 9.9 ) years. The characteristics of the two groups at baseline were similar and are detailed in Table 2.

\section{Treatment performance}

PGI-C scores were used to determine the percentage of responders versus nonresponders in the intramuscular and subcutaneous groups at days 7 and 14 (Figure 2). In both groups, the percentage of responders increased between days 7 and 14, with $77.7 \%$ (7/9) of the intramuscular group and $44.4 \%$ (4/9) of the subcutaneous group responding by day 14. Although there were more intramuscular responders at days 7 and 14, the final time point scores for the two methods were not significantly different $(P=0.335$, two-sided Fisher's Exact test, post hoc comparison).
The CGI-C scores were consistent with the PGI-C scores (Figure 2). In both the CGI-C and PGI-C assessments, the percentage of responders increased over time. Interestingly, the clinician assessment scored more responders, indicating more symptom improvement than the patient assessments.

The raw data scores for the PGI-C and CGI-C assessments are summarized in Tables $3 \mathrm{~A}$ and $3 \mathrm{~B}$. Overall, the data indicate an improvement in symptom exacerbation. Between days 7 and 14, both intramuscular and subcutaneous patients went from "minimally worse" or "much worse" to an improved symptom score. By day 14, the clinician assessment of "very much improved" or "much improved" increased to $100 \%$ for the intramuscular patients and $77.7 \%$ for the subcutaneous patients.

These data show that patients responded to a shorter, fiveday course of treatment, and that although the intramuscular route seemed more effective than the subcutaneous route, the difference was not statistically significant in this small sample.

Table 2 Demographics and clinical neurological measures at baseline

\begin{tabular}{lll}
\hline Parameter & $\begin{array}{l}\text { Intramuscular } \\
\text { group }(\mathbf{n}=10)\end{array}$ & $\begin{array}{l}\text { Subcutaneous } \\
\text { group }(\mathbf{n}=10)\end{array}$ \\
\hline $\begin{array}{l}\text { Age, mean (SD) } \\
\text { Gender, } \mathrm{n}(\%)\end{array}$ & $36.1(8.9)$ & $42.5(10.3)$ \\
$\quad$ Female & $7(70)$ & $10(100)$ \\
$\quad$ Male & $3(30)$ & 0 \\
Race, $n$ (\%) & & $10(100)$ \\
$\quad$ Caucasian & $10(100)$ & 7.3 \\
$\begin{array}{l}\text { Mean timed } \\
\text { walk test (seconds) }\end{array}$ & 6.9 & 14.5 \\
$\begin{array}{l}\text { Nine-hole Peg Test } \\
\text { Visual analog scale }\end{array}$ & 14.7 & 4.3 \\
EDSS & 6.3 & 2.50 \\
\hline
\end{tabular}

Abbreviations: SD, standard deviation; EDSS, Expanded Disability Status Scale. 
A

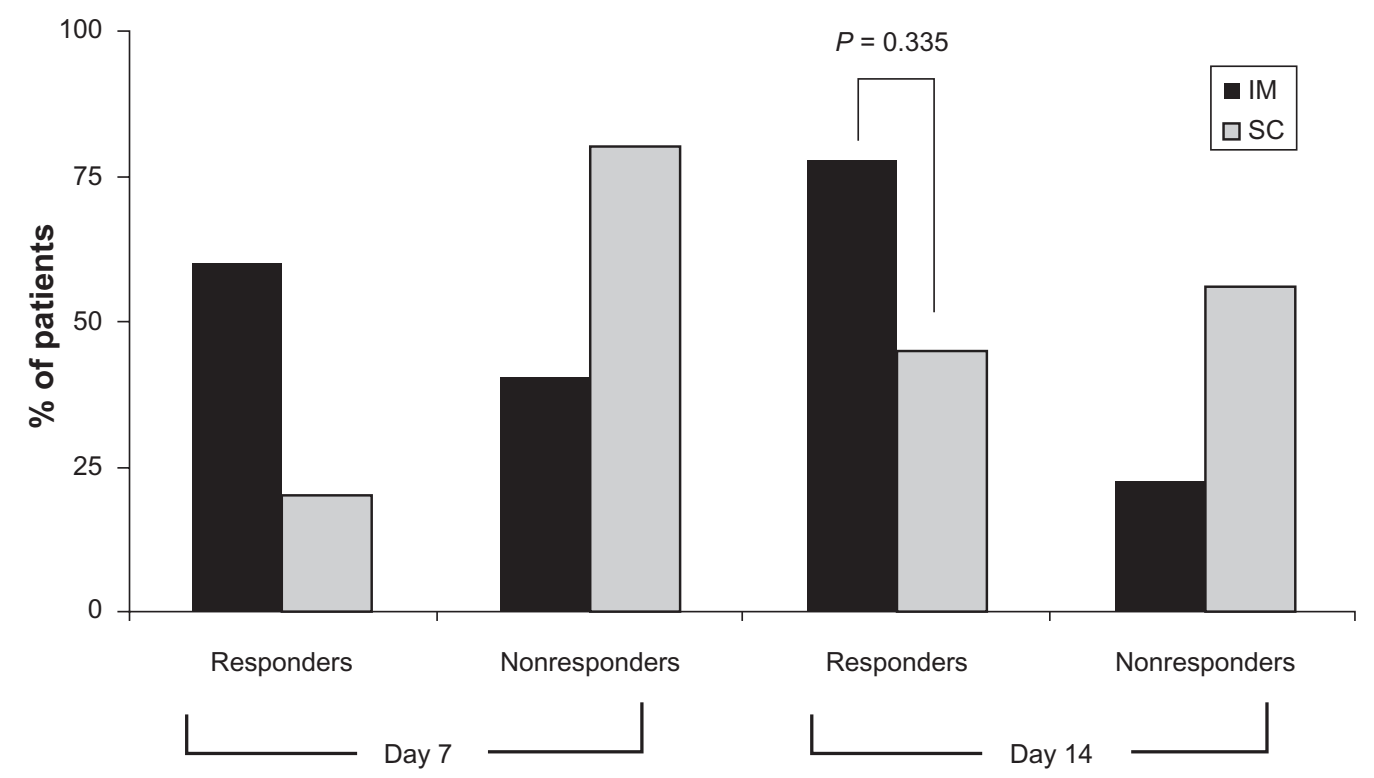

B

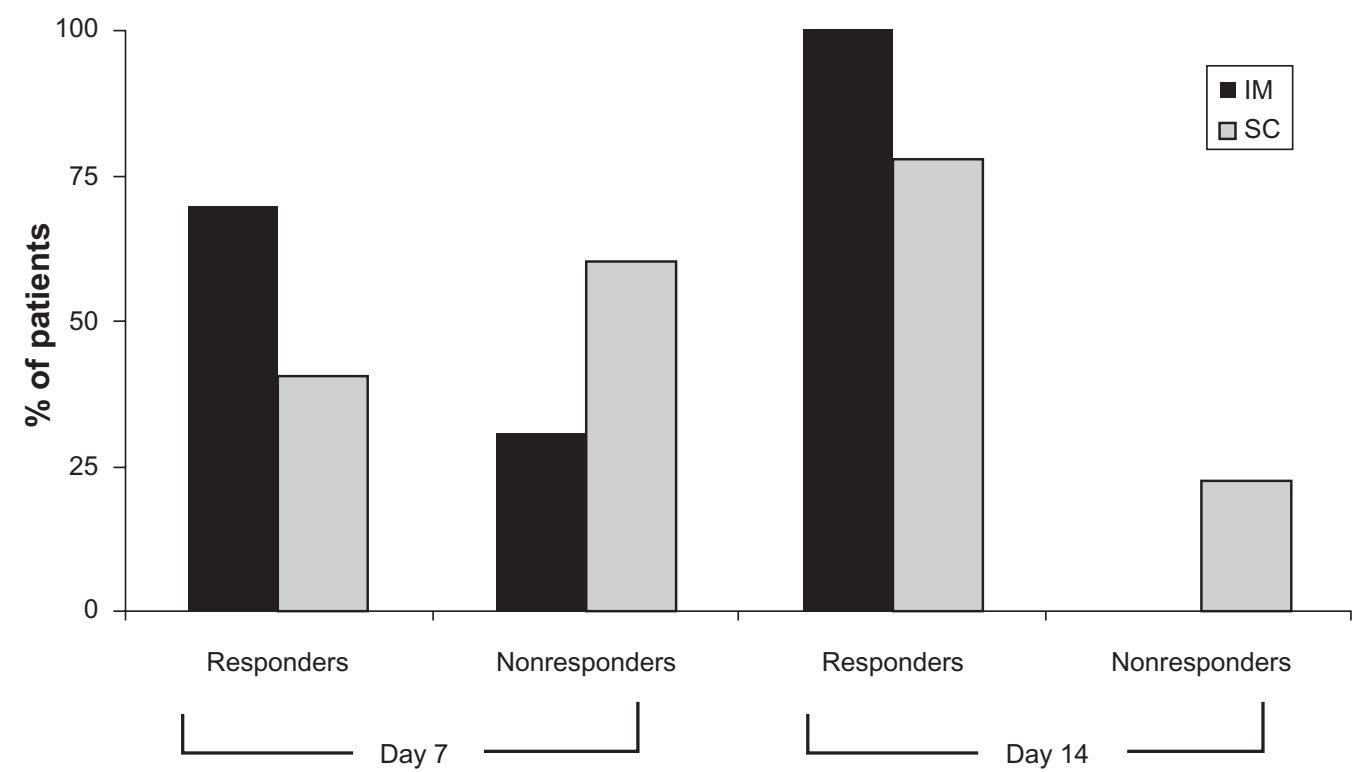

Figure 2 Percentage of responders as determined by the Patient Global Impression of Change (A) and Clinical Global Impression of Change (B) score cards on days 7 and I4. Responders were defined by a patient or clinical response of "much improved" or "very much improved." Both assessments reported a drug response to a five-day treatment in both the IM and SC groups, and an increase in drug response at day 14.

Abbreviations: CGI, clinical global impression of change; IM, intramuscular; SC, subcutaneous.

Table 3A Patient Global Impression of Change scores on days 7 and I4

\begin{tabular}{|c|c|c|c|c|c|c|}
\hline Study day & $\begin{array}{l}\text { Very much } \\
\text { improved* }\end{array}$ & $\begin{array}{l}\text { Much } \\
\text { improved* }\end{array}$ & $\begin{array}{l}\text { Minimally } \\
\text { improved }\end{array}$ & $\begin{array}{l}\text { No } \\
\text { change }\end{array}$ & $\begin{array}{l}\text { Minimally } \\
\text { worse }\end{array}$ & $\begin{array}{l}\text { Much } \\
\text { worse } \\
\end{array}$ \\
\hline \multicolumn{7}{|c|}{ Intramuscular group $(n=10)^{\dagger}$} \\
\hline Day 7 & $10 \%(1)$ & $50 \%(5)$ & $20 \%(2)$ & $10 \%(1)$ & $10 \%(1)$ & 0 \\
\hline Day 14 & $44.4 \%(4)$ & $33.3 \%(3)$ & 0 & $22.2 \%(2)$ & 0 & 0 \\
\hline \multicolumn{7}{|c|}{ Subcutaneous group $(n=10)^{\ddagger}$} \\
\hline Day 7 & $10 \%(1)$ & $10 \%(I)$ & $10 \%(1)$ & $20 \%(2)$ & $30 \%(3)$ & $20 \%(2)$ \\
\hline Day 14 & $11.1 \%(1)$ & $33.3 \%$ (3) & 0 & $33.3 \%$ (3) & $11.1 \%(I)$ & II.I\%(I) \\
\hline
\end{tabular}


Table 3B Clinical Global Impression of Change scores on days 7 and I4

\begin{tabular}{|c|c|c|c|c|c|c|}
\hline Study day & $\begin{array}{l}\text { Very much } \\
\text { improved* }\end{array}$ & $\begin{array}{l}\text { Much } \\
\text { improved* }\end{array}$ & $\begin{array}{l}\text { Minimally } \\
\text { improved }\end{array}$ & $\begin{array}{l}\text { No } \\
\text { change }\end{array}$ & $\begin{array}{l}\text { Minimally } \\
\text { worse }\end{array}$ & $\begin{array}{l}\text { Much } \\
\text { worse }\end{array}$ \\
\hline \multicolumn{7}{|c|}{ Intramuscular group $(n=10)$} \\
\hline Day 7 & $60 \%(6)$ & $10 \%(I)$ & $20 \%(2)$ & $10 \%(1)$ & 0 & 0 \\
\hline Day 14 & $90 \%(9)$ & $10 \%(I)$ & 0 & 0 & 0 & 0 \\
\hline \multicolumn{7}{|c|}{ Subcutaneous group $(n=10)^{\ddagger}$} \\
\hline Day 7 & $30 \%(3)$ & $10 \%(1)$ & $40 \%(4)$ & $20 \%(2)$ & & \\
\hline Day 14 & $44.4 \%(4)$ & $33.3 \%$ (3) & II.1\% (I) & $11.1 \%(1)$ & 0 & 0 \\
\hline
\end{tabular}

Notes: *Defined as responders; †Scores from nine patients were available for day 14 (the score from the absent intramuscular patient was "much improved" on day I3); ${ }^{\ddagger} \mathrm{n}=9$ on day $\mathrm{I} 4$ in the subcutaneous group (one patient discontinued the study); CGI-C was consistent with PGI-C. There were more responders in the intramuscular treatment group but no statistically significant differences in the percentage of responders between the two groups $(P=0.335$, two-sided Fisher's Exact test).

Abbreviations: CGI-C, Clinical Global Impression of Change; PGI-C, Patient Global Impression of Change.

The visual analog scale data (Figure 3) support the PGI-C data showing symptom improvement by day 14 in both the intramuscular and subcutaneous treatment groups. The intramuscular treatment group showed a numerically greater response than the subcutaneous treatment group $(+3.8$ units versus +1.4 units, respectively). The final improvement scores (day 14) for the two groups were very similar, ie, 2.5 (intramuscular) and 2.9 (subcutaneous), on a scale of 0-10, where $0=$ "no exacerbation symptoms" and $10=$ "worst exacerbation symptoms possible."

The objective measures of function from a mean timed walk and the Nine-hole Peg Test are presented in Table 4. There is some improvement seen in the mean timed walk and Nine-hole Peg Test scores, although neither was statistically or clinically significant. This may be expected, because baseline scores (day 1) were already in a highly functional range.

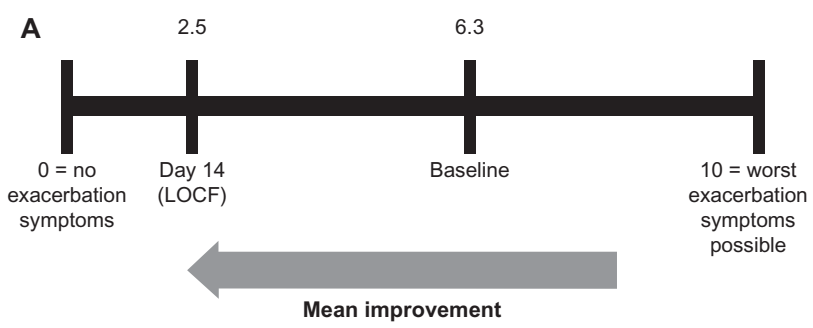

B

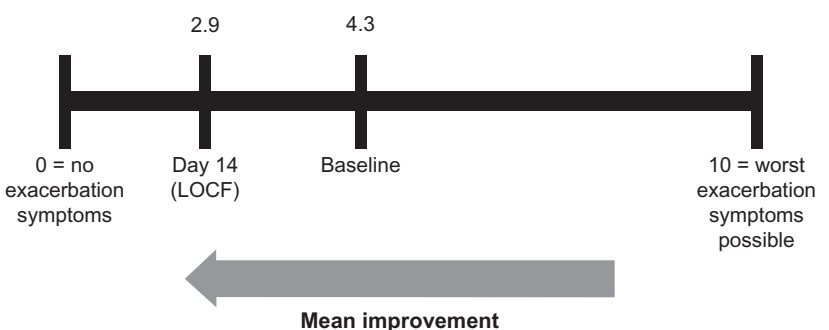

Figure 3 Mean improvement on the visual analog scale in the IM (A) and SC (B) groups. Comparable improvement of the symptoms of acute exacerbation was noted in both IM and SC groups on the patient visual analog scale.

Abbreviations: LOCF, last observation carried forward; SC, subcutaneous; IM, intramuscular.
The EDSS scores, measuring level of disability, are reported in Table 5. As for the other assessments, there was some ability improvement in both the intramuscular and subcutaneous treatment groups from visit 1 (baseline) to visit 3 (day 14). However, as in the objective measures of function, the baseline EDSS scores are already highly functional. The median scores at baseline in this patient population ranged from 2.3 to 2.8 , indicating "mild to moderate disability."

In all, the subjective assessments of a shorter five-day course of treatment showed good patient response and tolerability, regardless of route of administration. In the objective assessments of function, patients generally improved after treatment. However, the low initial baseline scores suggest that this cohort of patients retained a high level of ability at study enrollment.

Patients from both treatment groups rated the study treatment high for satisfaction, convenience, and tolerability on the drug attitude questionnaire on day 14 (Table 6). Of the seven patients $(70 \%)$ in the intramuscular treatment group who reported experiencing pain associated with injection, all but one patient described the pain as mild. In contrast, only $33.3 \%$ of patients in the subcutaneous group reported injection site pain, all of whom described the pain as mild. Of note, $95 \%$ of study patients who completed the questionnaire $(\mathrm{n}=18)$ reported that an injection at home was significantly more convenient than infusion in the clinic. Further, $90 \%$ of patients in the intramuscular group and $67 \%$ in the subcutaneous group said they would request the same intervention for any future acute exacerbations.

\section{Safety and tolerability}

Nineteen patients completed the study, and one patient in the subcutaneous group withdrew, citing lack of improvement. The injections were well tolerated by both treatment groups. Severe adverse events were reported in one intramuscular subject who complained of localized pain and numbness of 
Table 4 Results of a timed walk and the Nine-hole Peg Test (mean \pm standard deviation)

\begin{tabular}{|c|c|c|c|c|c|c|}
\hline \multirow[t]{2}{*}{ Injection } & \multicolumn{3}{|c|}{ Mean timed walk (seconds) } & \multicolumn{3}{|c|}{ Nine-hole Peg Test (pegs/second) } \\
\hline & Visit I & Visit 2 & Visit 3 & Visit I & Visit 2 & Visit 3 \\
\hline \multirow[t]{2}{*}{ Intramuscular } & $\mathrm{n}=10$ & $\mathrm{n}=10$ & $\mathrm{n}=10$ & $\mathrm{n}=10$ & $\mathrm{n}=10$ & $\mathrm{n}=10$ \\
\hline & $6.9 \pm 2.4$ & $6.3 \pm 1.3$ & $5.9 \pm 1.2$ & $14.7 \pm 2.3$ & $13.7 \pm 2.8$ & $13.8 \pm 2.2$ \\
\hline \multirow[t]{2}{*}{ Subcutaneous } & $\mathrm{n}=10$ & $\mathrm{n}=10$ & $n=9$ & $\mathrm{n}=10$ & $\mathrm{n}=10$ & $n=9$ \\
\hline & $7.3 \pm 4.8$ & $7.4 \pm 5.1$ & $8.1 \pm 6.8$ & $14.5 \pm 3.5$ & $14.0 \pm 3.5$ & $13.7 \pm 3.2$ \\
\hline
\end{tabular}

Table 5 Expanded disability status scale scores (mean \pm standard deviation)

\begin{tabular}{llll}
\hline Injection & $\begin{array}{l}\text { Visit I } \\
\text { (baseline) }\end{array}$ & $\begin{array}{l}\text { Visit 2 } \\
\text { (day 7 } \pm \text { I day) }\end{array}$ & $\begin{array}{l}\text { Visit 3 } \\
\text { (day I4 } \pm \text { I day) }\end{array}$ \\
\hline Intramuscular & $\mathrm{n}=10$ & $\mathrm{n}=10$ & $\mathrm{n}=10$ \\
& $2.30 \pm 0.54$ & $1.20 \pm 0.95$ & $1.10 \pm 0.88$ \\
Subcutaneous & $\mathrm{n}=10$ & $\mathrm{n}=10$ & $\mathrm{n}=9$ \\
& $2.80 \pm 1.3$ & $2.65 \pm 1.38$ & $2.89 \pm 1.64$ \\
All & $\mathrm{n}=20$ & $\mathrm{n}=20$ & $\mathrm{n}=19$ \\
& $2.55 \pm 1.01$ & $1.93 \pm 1.37$ & $1.95 \pm 1.55$ \\
\hline
\end{tabular}

the left hand and leg on one occasion and of the back and leg on another occasion. One subject in the subcutaneous treatment group had an ear infection. No serious adverse events were reported in either group.

\section{Discussion}

A recent comprehensive review of steroid hormone therapy for exacerbations of multiple sclerosis suggested that a short duration (five days) of methylprednisolone and a long duration (14 days) of ACTH appeared to have significant differences in treatment outcomes. ${ }^{7}$ We wanted to know if a short course of treatment (five days) using ACTH could still alleviate symptoms of an acute exacerbation of multiple sclerosis, and if patients liked the ACTH treatment protocol.
In this small pilot study, we examined, for the first time, if a shorter, self-administered five-day course of ACTH therapy would show a treatment response.

In the literature, clinical trial data on the use of ACTH in acute exacerbations of multiple sclerosis come from two placebo-controlled, double-blind studies and one doubleblind comparator trial (ACTH versus methylprednisolone) involving a total of 298 subjects across all studies. ${ }^{3-5}$ In a study by Miller et al, ACTH gel was dosed $60 \mathrm{U}$ intramuscularly twice daily in the first week, $40 \mathrm{U}$ twice daily in the second week, and then $60 \mathrm{U}, 40 \mathrm{U}$, and $20 \mathrm{U}$ on days 2,4 , and 6 of the third week. ${ }^{3}$ Rose et al dosed ACTH at $40 \mathrm{U}$ twice daily intramuscularly for seven days, tapered to $20 \mathrm{U}$ twice daily for four days, and then $20 \mathrm{U}$ daily for three days in their study. ${ }^{4}$ Thompson et al compared a regimen of intravenous methylprednisolone dosed $1 \mathrm{~g}$ /day for three days, with a 14-day course of ACTH given intramuscularly as follows: $80 \mathrm{U} /$ day for seven days, $40 \mathrm{U} /$ day for four days, and $20 \mathrm{U} /$ day for three days. ${ }^{5} \mathrm{~A}$ significant treatment effect of ACTH was demonstrated in the placebo-controlled studies, and no difference with methylprednisolone was reported in the small comparative study. Mild side effects were reported with $\mathrm{ACTH}$ and not methylprednisolone in the comparative study; it would be interesting to know if a

Table 6 Responses to the drug attitude questionnaire on visit 3

\begin{tabular}{|c|c|c|c|}
\hline Question & Possible responses & $\begin{array}{l}\text { Intramuscular } \\
\text { n (\%) }\end{array}$ & $\begin{array}{l}\text { Subcutaneous } \\
\text { n (\%) }\end{array}$ \\
\hline \multirow[t]{4}{*}{ Any pain associated with injection? } & No pain & $3(30)$ & $6(67)$ \\
\hline & Mild pain & $6(60)$ & $3(33)$ \\
\hline & Moderate pain & I (I0) & 0 \\
\hline & Significant pain & 0 & 0 \\
\hline Difficult to master the injection & No difficulty & $7(70)$ & $9(100)$ \\
\hline \multirow[t]{3}{*}{ technique? } & Minimal difficulty & $3(30)$ & 0 \\
\hline & Moderate difficulty & 0 & 0 \\
\hline & Significant difficulty & 0 & 0 \\
\hline Injection at home more convenient & Significantly more convenient & $10(100)$ & $8(89)$ \\
\hline \multirow[t]{3}{*}{ than infusions in clinic? } & Moderately more convenient & 0 & 0 \\
\hline & Minimally more convenient & 0 & 0 \\
\hline & No difference & 0 & $I(I I)$ \\
\hline Would you ask for this drug/route & Yes & $9(90)$ & $6(67)$ \\
\hline \multirow[t]{2}{*}{ for an acute exacerbation? } & Not sure & 0 & $\mathrm{I}(\mathrm{II})$ \\
\hline & No & $\mathrm{I}(\mathrm{I0})$ & $2(22)$ \\
\hline
\end{tabular}


shorter course of ACTH treatment would result in fewer side effects. No large studies have directly compared methylprednisolone with ACTH in the treatment of acute exacerbations of multiple sclerosis.

The gel formulation of ACTH is approved by the Food and Drug Administration for several different indications, given either intramuscularly or subcutaneously. ${ }^{6}$ There are limited data available on the duration, dosing, and route of administration of ACTH gel in the treatment of acute exacerbations of multiple sclerosis. This pilot study is the first attempt to compare ACTH gel therapy administered either intramuscularly or subcutaneously. We found that patients in both the intramuscular and subcutaneous treatment groups reported improvement in their condition after a five-day course of treatment with ACTH gel dosed at $80 \mathrm{U} /$ day. Improvement was also observed on the CGI-C scale, indicating that clinicians also observed meaningful improvements.

This was a pilot project and therefore had certain limitations. Because of the small sample size, random assignment to treatment arms may not have eliminated bias entirely. In addition, because there was no control arm, it is unknown what percentage of exacerbations would have resolved spontaneously. It was difficult to measure improvement objectively in this study, because the patients demonstrated good function at enrollment and the study period was short (14 days). Lastly, while the drug attitude questionnaire showed general patient satisfaction with the ACTH treatment, the questionnaire failed to determine the sources of satisfaction, such as drug efficacy, tolerability, convenience, or cost. It will be important to address these issues in larger trials based upon the results of this small pilot study.

If confirmed, a shorter protocol for treatment of acute exacerbations with ACTH could have implications for disease management and clinical practice as it moves treatment from the clinic and hospital setting to the home, giving patients more control over their disease and improving their quality of life. Shortening the treatment time may lessen the cost of using ACTH while maintaining clinical efficacy and increasing convenience to the patient, and changing the injection route from intramuscular to subcutaneous may minimize the pain and difficulty of treatment. Together, these elements of our modified protocol may decrease the burden of disease for the patient with multiple sclerosis, improve quality of life, and give physicians more patient-friendly options when treating acute exacerbations.

More clinical trials are needed to examine new dosing protocols with our "old" drugs as well as exploring drug combinations with the newer drugs available. However, the expense and time required for large, placebo-controlled studies can make this impractical. Small pilot studies may have value in such cases, where changes in drug protocol or special treatment protocols for patient subpopulations may be evaluated qualitatively and shared with the clinical community. In this way, we may be able to use the drugs we currently have more effectively.

\section{Conclusion}

Our study in 20 patients suggests that a five-day course of patient-administered ACTH gel therapy relieves symptoms of acute exacerbations of multiple sclerosis when administered either as intramuscular or subcutaneous injections. In most cases, the patient's lifestyle may be less disrupted by a self-administered injection compared with an infusion in the clinic, giving the patient more independence and control of their disease management. In addition, a shorter five-day course of treatment would reduce costs to the patient, in terms of drug cost, time, and the likelihood of adverse events. These results warrant further study of short-term, self-administered ACTH therapy for acute exacerbations of multiple sclerosis. Larger, placebo-controlled studies are needed to determine the optimal dose of ACTH gel, duration of treatment, and route of administration, as well as its role compared with steroid therapy.

\section{Acknowledgments}

The authors thank Rick Sipe for assistance with data collection, John Schulte of Austere Software for assistance with data analysis, and Mini Balaram and Tara Gupta of MedVal Scientific Information Services for assistance with manuscript preparation. Funding for this study and manuscript preparation was provided by an unrestricted grant from Questcor Pharmaceuticals. The authors did not receive payment for their work on this manuscript.

\section{Disclosure}

JPS has received consulting or advisory fees from Biogen Idec and EMD Serono and honoraria for speaking from Bayer, Biogen Idec, EMD Serono, and TEVA. CS has received consulting or advisory fees from Bayer, EMD Serono, Questcor, and TEVA, and honoraria for speaking from Bayer, EMD Serono, and TEVA. DMS has received honoraria for speaking from EMD Serono. Data from the study were presented in part at the annual meeting of the Consortium of Multiple Sclerosis Care Centers, May 31-June 3, 2006, Scottsdale, AZ. 


\section{References}

1. Ringold S, Lynm C, Glass RM. JAMA patient page. Multiple sclerosis. JAMA. 2006;296:2880.

2. National Institute of Neurologic Disorders and Stroke, National Institutes of Health. Multiple Sclerosis: Hope Through Research. Available from: http://www.ninds.nih.gov/disorders/multiple_sclerosis/detail_multiple_ sclerosis.htm\#158943215. Accessed February 22, 2011.

3. Miller H, Newell DJ, Ridley A. Multiple sclerosis. Treatment of acute exacerbations with corticotrophin (A.C.T.H.). Lancet. 1961;2: 1120-1122.

4. Rose AS, Kuzma JW, Kurtzke JF, Namerow NS, Sibley WA, Tourtellotte WW. Cooperative study in the evaluation of therapy in multiple sclerosis. ACTH vs placebo - final report. Neurology. 1970; 20:1-59.

5. Thompson AJ, Kennard C, Swash M, et al. Relative efficacy of intravenous methylprednisolone and ACTH in the treatment of acute relapse in MS. Neurology. 1989;39:969-971.

6. H.P. Acthar ${ }^{\circledR}$ Gel (repository corticotropin injection) [Prescribing information]. Union City, CA: Questcor Pharmaceuticals Inc; 2010.
7. Filippini G, Brusaferri F, Sibley WA, et al. Corticosteroids or ACTH for acute exacerbations in multiple sclerosis. Cochrane Database Syst Rev. 2000;4:CD001331.

8. Brod SA, Morales MM. Bio-equivalence of intramuscular and SQ H.P. Acthar Gel. Biomed Pharmacother. 2009;63:251-253.

9. Prettyman J. Subcutaneous or intramuscular? Confronting a parenteral administration dilemma. Medsurg Nursing. 2005;14:93-98.

10. Guy W. 053 SCL-90 self-report symptom inventory. In: $E C D E U$ Assessment Manual for Psychopharmacology Revised, 1976. Rockville, MD: DHEW Publication; 1976.

11. Guy W. 028 CGI clinical global impressions. In: ECDEUAssessment Manual for Psychopharmacology Revised, 1976. DHEW Publication; 1976.

12. Kurtzke JF. Disability rating scales in multiple sclerosis. Ann NY Acad Sci. 1984;436:347-360.

13. Collen FM, Wade DT, Bradshaw CM. Mobility after stroke: Reliability of measures of impairment and disability. Int Disabil Stud. 1990;12: 6-9.

14. Oxford Grice K, Vogel KA, Le V, Mitchell A, Muniz S, Vollmer MA. Adult norms for a commercially available Nine Hole Peg Test for finger dexterity. Am J Occup Ther. 2003;57:570-573.

\section{Publish your work in this journal}

Drug Design, Development and Therapy is an international, peerreviewed open-access journal that spans the spectrum of drug design and development through to clinical applications. Clinical outcomes, patient safety, and programs for the development and effective, safe, and sustained use of medicines are a feature of the journal, which has also been accepted for indexing on PubMed Central. The manuscript management system is completely online and includes a very quick and fair peer-review system, which is all easy to use. Visit http://www.dovepress.com/testimonials.php to read real quotes from published authors.

Submit your manuscript here: http://www.dovepress.com/drug-design-development-and-therapy-journal 